\title{
Efficacy of the mini mesh for reducing prolapse recurrence: comparison of two implant positioning methods
}

\author{
(D) AYELLET NEUMANN KOREN ${ }^{1}$, (1) MENAHEM NEUMAN ${ }^{1,2}$, (1) JACOB BORNSTEIN ${ }^{1,2}$ \\ 1Bar Ilan University, Azrieli Faculty of Medicine, Safed, Israel \\ 2Department of Obstetrics and Gynaecology, Galilee Medical Center, Nahariya, Israel
}

\begin{abstract}
Objective: We aimed to compare the postoperative complications, cure rates, and patient satisfaction in mini mesh implantation with and without anterior arm fixation to the para vesical fascia for the re-enforcement of the anterior pelvic floor compartment.

Materials and Methods: Thirty female patients diagnosed with symptomatic stage 3 pelvic organ prolapse (POP) of the anterior pelvic floor compartment were operated on by a single surgeon, using the SERATOM PA MR MN mini mesh graft (Serag-Wiessner, Naila, Germany). In thirty other women, serving as a control group, the anterior arms were positioned without fixation, while in the study group anterior arm fixation was added. Surgery complications, patients' satisfaction 3 months after the surgery, POP grade and volume of prolapse, and subjective variables such as a feeling of bulge, pain, and dyspareunia were compared between the two groups.
\end{abstract}

Results: Significant anatomical and functional improvements were documented in both groups with respect to the objective $(\mathrm{p}<0.05)$ and subjective $(p<0.01)$ criteria, except for pain, dyspareunia, and fecal incontinence. All patients reported high satisfaction.

Conclusion: Surgery with and without fixation led to significant improvement.

Keywords: Mini mesh; pelvic floor reconstruction; pelvic organ prolapse

\section{INTRODUCTION}

Pelvic organ prolapse (POP) is a common disorder in women during the reproductive, menopausal, and post-menopausal periods; it is estimated that $50 \%$ of all parous women will have an anatomical POP. POP impairs urinary, bowel, and sexual functions as well as self-esteem. While herniation of the anterior pelvic floor compartment is associated with cystocele, that of the posterior pelvic floor compartment is associated with rectocele, enterocele, and uterine prolapse. Approximately $11 \%$ of all women are surgically treated for symptomatic POP. However,
$30 \%-65 \%$ of the surgically treated patients require repeat prolapse surgery. ${ }^{1}$

Owing to possible complications, the use of a mesh implant in the treatment of POP is controversial. To support the internal pelvic organs, the weakened pelvic fascia is replaced by the synthetic mesh. 2,3 This has been previously shown to be effective and safe with the posterior intra-vaginal slingplasty (PIVS) in the treatment of vaginal apex prolapse. The invasiveness and painful complications of old surgical techniques encouraged Petros et al. ${ }^{4}$ to design this new treatment for anatomical restoration. As a forerunner to future mesh design and surgical techniques, the

Address for Correspondence: Jacob Bornstein, Bar Ilan University, Azrieli Faculty of Medicine, Safed, Israel and Department of Obstetrics and Gynecology, 
PIVS graft is solely a sling, secured at two ends to the uterosacral ligaments. ${ }^{5}$ As will be discussed later, reconstructive POP surgeries frequently involve the implantation of large meshes with wide and deep pelvic dissection; in addition, it bears some inherent hazards due to these implants and the dissection involved. ${ }^{2}$ Furthermore, the mesh is secured in place by suturing the mesh arms to the sacrospinous ligament (SSL) and also from the bladder neck to the uterine cervix to achieve anterior compartment support.

Intraoperative complications of vaginal meshes include accidental blood vessel damage, haemorrhage, and visceral (bladder and intestinal) and neural injuries. The postoperative complications include hematomas and infections, overactive bladder (OAB) symptoms, stress urinary incontinence (SUI), vaginal and pelvic pain, and dyspareunia. ${ }^{3,5,6}$

Since vaginal implanted meshes are only covered by the thin vaginal wall (mucosa and underlying fascia) and are also subjected to constant pressure, postoperative mesh displacement and its exposure is troubling and a widespread problem. The mini mesh maintains therapeutic outcomes and reduces the risk of tissue trauma and mesh-related complications by reducing the implant size (being $75 \%$ less than the original mesh size) and the number of fixation points. ${ }^{3,7}$

In 2011 and 2008, the Food and Drug Administration (FDA) issued a warning regarding the POP repair technique stating serious adverse events are neither rare nor mild; however, the use of mesh in this POP repair technique does not conclusively improve clinical outcomes. Furthermore, the FDA ordered a continuous evaluation of the effects of using surgical mesh and also noted the need for clinical studies in the field of urogynecology. ${ }^{8}$ As a result, the mini mesh was developed to overcome and minimize serious adverse events without compromising effectiveness. Later on, in 2019 the FDA issued an order that remaining manufacturers of transvaginal mesh for apical/anterior compartment prolapse stop selling and distributing their products. ${ }^{9}$

Currently, many of the grafts used in the reconstruction POP are mini meshes, as they have a lower recurrence rate and also simultaneously reduce mesh-related complication rates and severity. ${ }^{2,7}$ The mesh examined in this study, augmenting POP repair operation, is similar to that used in the previously reported PIVS technique in terms of positioning and lower mesh size (unlike the large meshes used thereafter). It is currently unclear whether the anterior mini mesh arms should be fixated para vesical or only positioned without fixation. There is a clear lack of evidence-based studies and treatment decisions are largely based on the surgeon's experience and training. This research compares surgical complications and patient satisfaction between patients with fixated mini mesh arms and those only positioned without fixation. We hypothesized that non-fixated anterior mini mesh arms are inferior to the fixated ones, as the non-fixated mesh might not stay in place, leading to an impairment in an appropriate anterior pelvic floor compartment reinforcement and a consecutive cystocele formation, thereby necessitating further corrective operations.

\section{MATERIALS AND METHODS}

This was a retrospective, cohort, non-randomized, non-blinded, double arm and dual center study (performed by a single surgeon) involving female patients diagnosed with advanced symptomatic stage 3 anterior POP; the surgeries were performed between 2017 and 2018. The first 30 patients (the control group) were operated using non-fixated mesh arms positioned para vesical, and the subsequent 30 patients (the study group) were operated with para vesical mesh arm fixation to the facia on both sides of the bladder cervix. All patients operated upon during this period were included in the study. Patients with incomplete records were excluded from this study.

All patients received first generation cephalosporin antibiotics intravenously half an hour before surgery, followed by an iodine antiseptic vaginal and surgical field wash. All surgeries were conducted under general anaesthesia. Prior the first surgical incision, $50 \mathrm{ml}$ of saline was injected at the midline of the anterior vaginal wall followed by a longitudinal incision, subfascial lateral dissection toward the pelvic sidewall until the ischiatic spine and then to the mid-portion of the SSL, to which the mesh arms would be fixated to. The other arms were passed through the obturator membrane and those from the bladder neck to the uterine cervix. The two surgical groups differed with respect to the mesh anterior arms sutured (study group) or positioned (control). After the mini mesh was placed and fixated, the vaginal wall was re-sutured in two layers: first the fascia and then the mucosa with running absorbable sutures.

Pre- and postoperative data were obtained for all patients from the medical records and were tabulated and evaluated. According to the instructions for conducting clinical research involving human subjects, this study was approved by the Institutional Research Board (Helsinki committee) at the Galilee Medical Center. Authorization number 0008-18-NHR, on April $11^{\text {th }}$, 2018. All data collected from the medical records were stored anonymously.

Participants of both groups were treated using the same mini mesh implant, SERATOM PA MR MN ${ }^{\circledR}$ (Serag-Wiessner, Naila, Germany). Furthermore, patients who were diagnosed with 
posterior vaginal wall relaxation were concomitantly subjected to posterior colporrhaphy with or without mesh augmentation. Moreover, patients with urinary stress incontinence underwent additional anti-incontinence surgery with sub-mid-urethral slings (SMUS), using TVT- Obturator ${ }^{\circledR}$, TVT-Abbrevo ${ }^{\circledR}$ (Gynecare, Somerville, USA) or Serasis ${ }^{\circledR}$ (Serag-Wiessner, Naila, Germany). Patients were discharged after overnight hospitalization and were followed up a day, a month, and 3 months later. ${ }^{10}$ All procedures were performed by the same surgeon (M.N.).

The age, obstetric history, comorbidities (e.g. hypertension, diabetes mellitus (DM) type 2, gastric esophageal reflux and cardiovascular disease), family history of POP, self-reported urinary tract infection history, and information regarding the previous hysterectomy, POP, or SUI repair were all compared. Furthermore, a physical evaluation was performed early postop and one-month post-op by the surgeon. Subsequently, objective and subjective variables were measured and recorded pre-operatively and one-month postoperatively. The objective variables included POP severity grade, ${ }^{10}$ and volume of prolapse, an objective clinical estimation of the surgeon regarding tissue volume in millilitres protruding out of the vagina.

Additionally, a new measurement of POP severity was proposed; advanced prolapse has been defined as one that protrudes 0.5 litre (L) and over, out of the vagina. Subjective variables included various complaints such as bulge, pain, dyspareunia, voiding problem, OAB symptoms, SUI, UTI, fecal incontinence, and constipation. Information obtained on the operative and postoperative days were included in the operational remarks; complications such as rash and itching, groin pain, fever $(>38.5$ $\left.{ }^{\circ} \mathrm{C}\right)$, and need for catheterization as well as patient's reported satisfaction 3 months post-operation were recorded.

\section{Statistical analysis}

In addition to descriptive statistical methods [mean \pm standard deviation (SD)], Student's t-test and Mann-Whitney $U$ test were used to identify potential differences between the two independent groups of parameters, with and without normal distributions, respectively. Variance analysis of intergroup values was performed using the Kruskal-Wallis test. Additionally, a oneway analysis of variance (ANOVA) test was used for normally distributed data. Spearman's rank correlation coefficient was used to determine potential correlations between multiple groups. The qualitative data were compared using the chisquare test. The results were considered statistically significant based on the 95\% confidence interval, and $p$-values less than 0.05. Z proportion test were used to evaluate the differences between the rates of complications in the literature and those in this study. In total, 56 patients were required to obtain $80 \%$ actual power of the study.

\section{RESULTS}

In total, 60 patients underwent reconstructive POP surgery for the anterior pelvic floor compartment between July $17^{\text {th }}$, 2017, and January $5^{\text {th }}, 2018$; the mean age was 66 years (SD: \pm 8.77 ; age range: $41-88$ years) and most of the patients were multiparous with a mean of 3.5 labours (SD: \pm 1.41 ; range: $1-8$ labours). In addition, $13.3 \%$ of the patients $(n=8)$ had a history of hysterectomy and $16.7 \%(n=10)$ of them had repeated POP surgeries: colpo-sacro-pexy $(n=8,13.3 \%)$, colporrhaphy $(n=1$, 1.7\%) and laparoscopic Burch colpo-suspension ( $n=1,1.7 \%$ ). Moreover, $75 \%$ of the patients had comorbidities: hypertension $(n=19,31.7 \%)$, DM $(n=9,15 \%)$, and cardiovascular disease $(n=3$, $5 \%)$. No significant statistical difference in these parameters was found between the two groups. Furthermore, records of familial history of POP was insufficient; therefore, no statistical conclusions could be drawn regarding this potential variable.

Significant statistical improvement $(p<0.05$ and $p<0.01)$ was observed in both groups for most of the objective and subjective criteria (Table 1), except for pain, dyspareunia, and fecal incontinence (all mild).

Two patients (3.3\%) reported minimal post-op pain; one patient reported one-month post-op groin pain and another reported pain due to a vaginal scar granulation (VAS 1-3). Dyspareunia and faecal incontinence could not be evaluated owing to poor data quality. Of four patients (6.6\%) with pre-op dyspareunia, two reported post-op improvement, while the other two had no sexual intercourse during the survey time. Furthermore, of the four patients suffering from faecal incontinence, three reported improvement, which was not statistically significant.

In the control group (para vesical mesh positioning without fixation), all patients reported significant improvement and a complete resolution of symptomatic voiding problems, which was statistically significant $(p<0.05)$. On the contrary, the improvement showed by the study group (para vesical mesh fixation to the fascia) was statistically insignificant.

Improvement in both $\mathrm{Ba}$ and $\mathrm{Bp}$ measurements (objective anterior and posterior POP-Q points $)^{9}$ was shown to be statistically significant $(p<0.01)$ for both groups, using the $Z$ test (Table 1). Using the Mann-Whitney $U$ test, the statistical significance $(p<0.05)$ of the delta Ba improvement was repeatedly demonstrated for the study group, compared to the control group (Table 2).

In total, seven patients (11.7\%) from both groups had very advanced prolapse (all had their vaginas bulging out, greater than $0.5 \mathrm{~L}$ ) and were also prone to complications and recurrence. ${ }^{11}$ Five patients (8.3\%) were diagnosed with $0.5 \mathrm{~L}$, one patient $(1.7 \%)$ 
Table 1. Improvement in objective and subjective measurements for the study and control groups

\begin{tabular}{|c|c|c|c|c|c|c|}
\hline & Z & SD & Mean & Z & SD & Mean \\
\hline Bulge pre-op & \multirow{2}{*}{$5.47^{* *}$} & 0.0 & 3.0 & \multirow{2}{*}{$5.39^{* *}$} & 0.0 & 3.0 \\
\hline Bulge post-op for 1 month & & 0.0 & 0.0 & & 0.2 & 0.0 \\
\hline Pain pre-op & \multirow{2}{*}{0.00} & 0.0 & 0.0 & \multirow{2}{*}{1.4} & 0.0 & 0.0 \\
\hline Pain post-op for 1 month & & 0.0 & 0.0 & & 0.3 & 0.1 \\
\hline Dyspareunia pre-op & \multirow{2}{*}{1.0} & 0.4 & 0.1 & \multirow{2}{*}{1.3} & 0.8 & 0.3 \\
\hline Dyspareunia post-op for 1 month & & 0.0 & 0.0 & & 0.4 & 0.1 \\
\hline Pre-op voiding problem & \multirow{2}{*}{1.84} & 0.7 & 0.3 & \multirow{2}{*}{$2.85^{*}$} & 1.2 & 0.8 \\
\hline 1-month post-op voiding problem & & 0.0 & 0.0 & & 0.0 & 0.0 \\
\hline Pre-op OAB Symptoms & \multirow{2}{*}{$3.81^{* *}$} & 0.9 & 1.1 & \multirow{2}{*}{$3.48^{* *}$} & 1.1 & 1.7 \\
\hline 1-month post-op OAB Symptoms. & & 0.4 & 0.1 & & 1.0 & 0.8 \\
\hline SUI pre-op & \multirow{2}{*}{$2.17^{*}$} & 1.3 & 0.8 & \multirow{2}{*}{$3.46^{* *}$} & 1.5 & 1.2 \\
\hline SUI post-op for 1 month & & 0.6 & 0.2 & & 0.0 & 0.0 \\
\hline Faecal incontinence pre-op & \multirow{2}{*}{1.41} & 0.6 & 0.2 & \multirow{2}{*}{1.41} & 0.5 & 0.1 \\
\hline 1-month faecal incontinence post-op & & 0.2 & 0.0 & & 0.0 & 0.0 \\
\hline Constipation pre-op & \multirow{2}{*}{$3.41^{* *}$} & 0.8 & 0.6 & \multirow{2}{*}{$2.73^{*}$} & 0.7 & 0.4 \\
\hline Constipation post-op for 1 month & & 0.0 & 0.0 & & 0.0 & 0.0 \\
\hline Ba pre-op & \multirow{2}{*}{$4.77^{* *}$} & 1.6 & 3.2 & \multirow{2}{*}{$4.82^{* *}$} & 1.2 & 3.6 \\
\hline Ba post-op for 1 month & & 0.4 & -2.7 & & 0.6 & 1.9 \\
\hline Bp pre-op & \multirow{2}{*}{$4.75^{* *}$} & 1.6 & 1.0 & \multirow{2}{*}{$4.74^{* *}$} & 1.7 & 1.0 \\
\hline 1-month Bp post-op & & 0.7 & -2.6 & & 0.9 & 2.4 \\
\hline C pre-op & \multirow{2}{*}{$4.79^{* *}$} & 4.1 & 2.1 & \multirow{2}{*}{$4.79^{* *}$} & 3.8 & 1.7 \\
\hline 1-month C post-op & & 0.6 & -5.4 & & 0.6 & 4.8 \\
\hline POP degree pre-op & \multirow{2}{*}{$5.39^{* *}$} & 0.0 & 3.0 & \multirow{2}{*}{$5.39^{* *}$} & 0.0 & 3.0 \\
\hline POP degree post-op for 1 month & & 0.2 & 1.0 & & 0.2 & 1.0 \\
\hline \multicolumn{7}{|c|}{$\begin{array}{l}{ }^{*} p<0.05,{ }^{* *} p<0.01 \\
\text { POP: Pelvic organ prolapse; study group: Para vesical mesh fixation to the fascia; control group: Para vesical mesh positioning; Pre-op: Pre-operative; } \\
\text { Post-op: Post-operative; OAB: Overactive bladder; SUI: Stress urinary incontinence; Ba: The 2/3 point at the anterior vaginal wall; Bp: The 2/3 point at the } \\
\text { posterior vaginal wall; SD: Standard deviation; C: Uterine cervix }\end{array}$} \\
\hline
\end{tabular}

with $0.75 \mathrm{~L}$, and another (1.7\%) with $1.0 \mathrm{~L}$ volume bulging out of the vaginal opening. These patients showed improvements in the objective criteria at the one-month postoperative check-up in the clinic; however, six patients still had first-degree POP and one had second-degree POP. No statistical interpretation could be drawn from these data.

Nine patients (15\%) had pre-op urinary tract infections (UTI) and three (5\%) had post-op UTI. Surgical field fibrosis or the exposure of previous mesh was noted in three patients (5\%). Fortyone patients (68.3\%) had concomitant anterior and posterior repairs (APR, colporrhaphies), whereas nineteen (31.7\%) had both APR and SMUS for SUI. Furthermore, five patients suffered intraoperative bleeding, resulting in a 1.5-3 gr (\%) decrease in $\mathrm{Hgb} ; 91.7 \%$ of all patients $(n=55)$ had less than $1 \mathrm{gr}(\%)$ change in delta haemoglobin (Hgb) (Table 3).
In the control group, two patients (1.3\%, respectively) had $2 \mathrm{gr}(\%)$ and $3 \mathrm{gr}(\%)$ decrease in delta $\mathrm{Hgb}$, respectively. Moreover, one (1.3\%) and two patients (2.7\%) from the study group had $1.5 \mathrm{gr}$ (\%) and $2 \mathrm{gr}(\%)$ decrease in delta Hgb, respectively. Similarities were observed between the results comparing the delta $\mathrm{Hgb}$ with those of patients who underwent additional APR and APR with trans vaginal tape surgeries (Table 4).

In addition, three APR patients (5\%) showed a decrease of $2 \mathrm{gr}$ (\%) in the Hgb levels and two APR + SMUS patients (2.7\%) showed 1.5 gr (\%) decrease. The chi-square test showed no statistical significance associated with the changes in the Hgb levels and the surgical technique used.

Early post-op remarks (one-month follow-up) included three patients (5\%) who developed vaginal scar granulation, which was treated with fulguration. On the contrary, late post-op remarks (three-month follow-up) included anticholinergic $(n=6,10 \%)$ 
Table 2. Comparison of subjective and objective measurements between groups

\begin{tabular}{|c|c|c|c|c|c|c|c|c|c|c|c|}
\hline \multirow{3}{*}{ Mann-Whitney U } & \multicolumn{5}{|c|}{ The study group } & \multicolumn{5}{|c|}{ The control group } & \\
\hline & \multicolumn{3}{|c|}{ Percentiles } & \multirow{2}{*}{ SD } & \multirow{2}{*}{ Mean } & \multicolumn{3}{|c|}{ Percentiles } & \multirow{2}{*}{ SD } & \multirow{2}{*}{ Mean } & \\
\hline & 75 & 50 & 25 & & & 75 & 50 & 25 & & & \\
\hline 420 & 0.0 & 0.0 & 0.0 & 0.0 & 0.0 & 0.0 & 0.0 & 0.0 & 0.3 & 0.1 & $\triangle$ Pain \\
\hline 406.5 & 0.0 & 0.0 & 0.0 & 0.4 & 0.1 & 0.0 & 0.0 & 0.0 & 0.5 & 0.0 & $\Delta$ Dyspareunia \\
\hline 431 & 0.0 & 1.0 & 2.0 & 1.0 & 1.0 & 0.0 & 1.0 & 2.0 & 1.1 & 0.9 & $\triangle \mathrm{OAB}$ symptoms \\
\hline 363 & 0.0 & 0.0 & 3.0 & 1.6 & 0.6 & 0.0 & 0.0 & 3.0 & 1.5 & 1.2 & $\Delta$ SUI \\
\hline 450 & 0.0 & 0.0 & 0.0 & 0.5 & 0.1 & 0.0 & 0.0 & 0.0 & 0.5 & 0.1 & $\Delta$ Faecal incontinence \\
\hline 380.5 & 0.0 & 0.0 & 1.0 & 0.8 & 0.6 & 0.0 & 0.0 & 1.0 & 0.7 & 0.4 & $\Delta$ Constipation \\
\hline
\end{tabular}

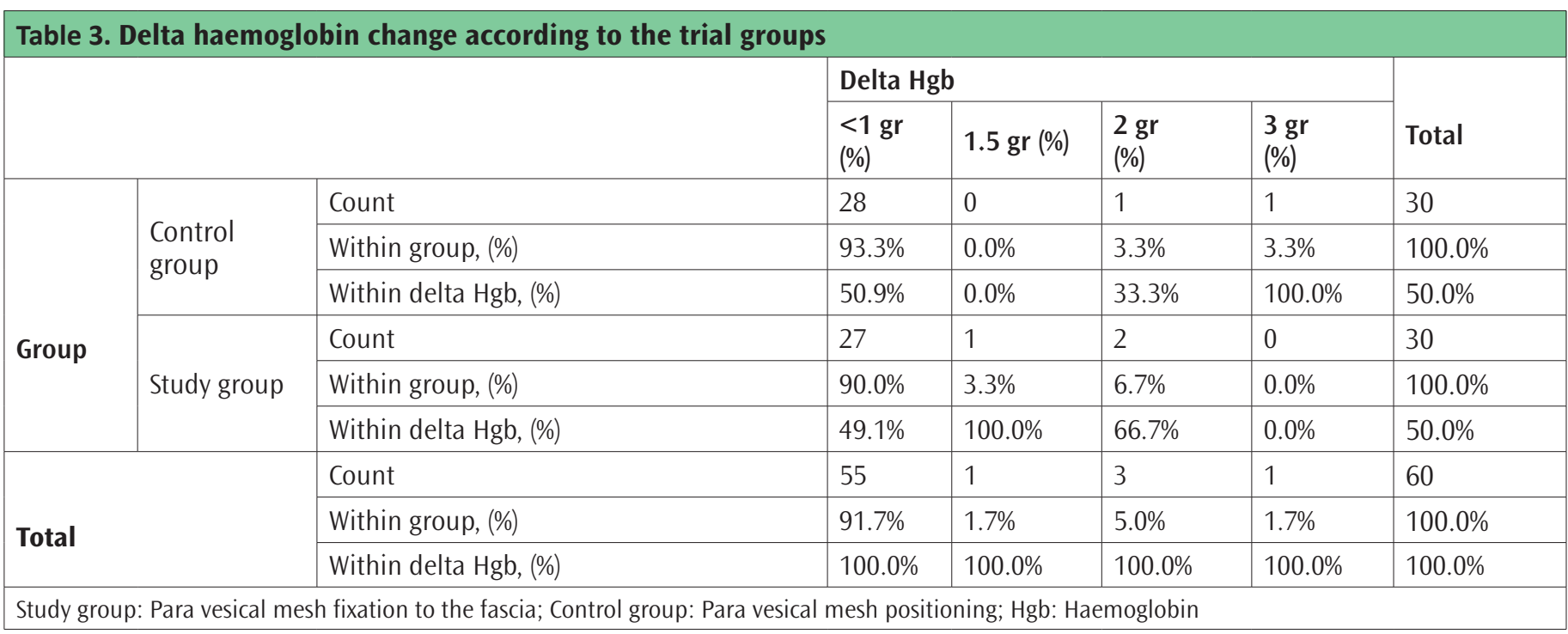

and beta-2 receptor agonists used $(n=1,1.7 \%)$ in the treatment of pre-operative OAB symptoms. In addition, sub-mid-urethral sling implantation was used for the treatment of de novo or aggravated SUI $(n=3,5 \%)$; one patient $(1.7 \%)$ reported minimal coccygeal pain.

Although no statistically significant conclusion was reached, firstday post-op complications were analysed. Subsequently, fiftyfour patients (90\%) had not suffered any post-op complications. Of all patients in the study group, one (1.3\%) needed a 2-day bladder catheterization, another (1.3\%) had self-limited mild left groin pain for two weeks; in addition, one patient (1.3\%) had mild self-limited perineal rash and itching. However, of all patients in the control group, two (2.7\%) needed bladder catheterization for 2 days, one (1.3\%) had self-limited fever of unknown origin with a body temperature of $38.5^{\circ} \mathrm{C}$ for 2 days and another (1.3\%) had mild self-limited left groin pain.

Satisfaction was assessed at the 3-month follow-up (Figure 1). Most of the patients ( $n=51,85 \%)$, including $90 \%$ of the patients in the study group ( $n=27)$ and $80 \%$ of the patients in the control group $(n=24)$, rated their overall satisfaction as maximal (Figure 1). Eight patients (13\%) gave the medium score and only one patient from the control group was dissatisfied and subsequently gave the lowest satisfaction score.

\section{DISCUSSION}

The major finding of this study is that both para vesical mesh positioning and para vesical mesh arm fixation can improve most objective and subjective parameters, thereby offering high 
Table 4. Delta haemoglobin changes according to the surgery performed

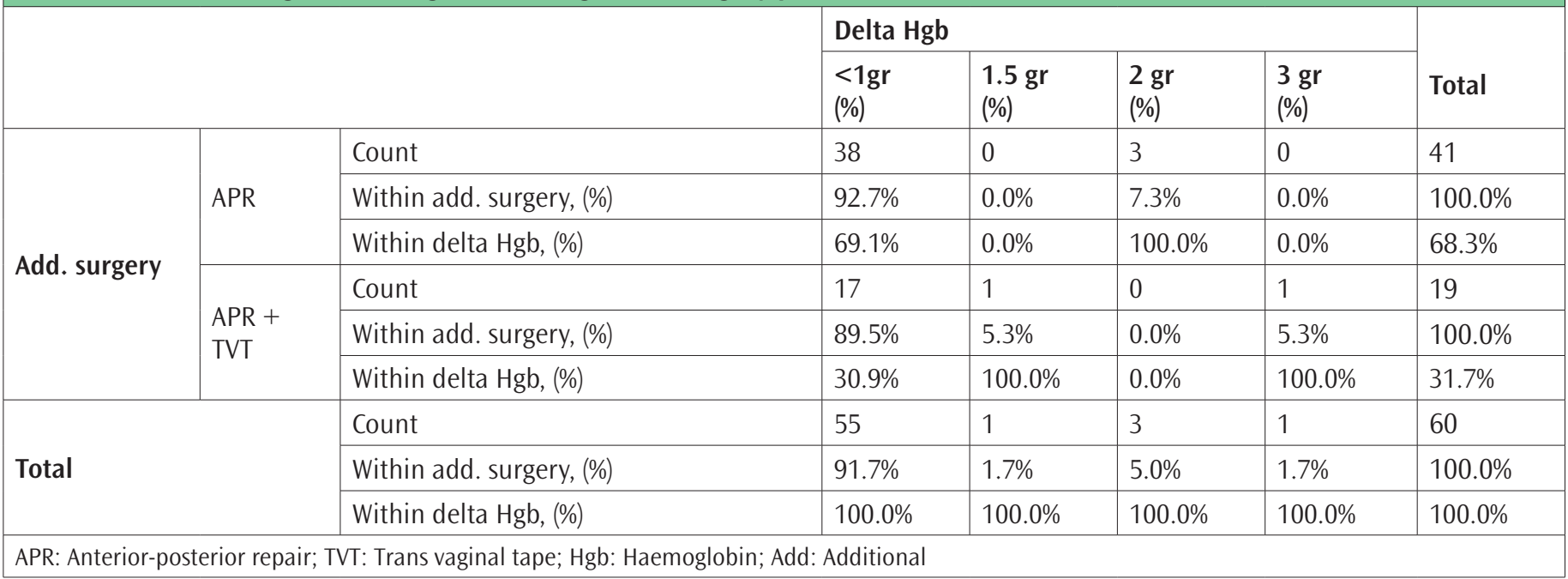

postoperative satisfaction, which was slightly higher in the study group. These results support previous studies, showing that desired outcomes can be achieved using the mini mesh.

Furthermore, the results suggest that "anterior mesh arm fixation" patients had better Ba position (the 2/3 point at the anterior vaginal wall according to the POP-Q) reconstructive outcomes. These slightly better outcomes could be attributed to the nature of the operational procedure of the study group (where mesh was fixated to the para vesical); thus, reinforcing the anatomical structure by keeping the vaginal wall in place. Improvements in either group are of limited significance owing to the relatively small group size.

Both groups showed improved subjective voiding: patients reported no post-operative voiding complaints. Statistical significance was demonstrated in the control group, where all 10 patients with pre-operative voiding had their problems fully resolved. Because the study group had fewer cases (only four patients with pre-op voiding complaints: all of which were resolved), no statistically significant conclusions could be drawn from these data.

Furthermore, no statistically significant differences could be observed for pain, dyspareunia, granulation tissue formation, and faecal incontinence, owing to poor data quality.

Although patients should be informed that anticholinergic medications might be needed for de novo or worsening OAB symptoms, SMUS implantation for SUI, or painkillers for buttock pain, it was noted that the vast majority of patients enjoyed the benefits of mini mesh POP reconstructions with no concerns or complaints.

Moreover, $85 \%$ of the patients (90\% of the study group and $80 \%$ of the control group) were highly satisfied with the procedure. In addition, eight patients comprising five from the control group

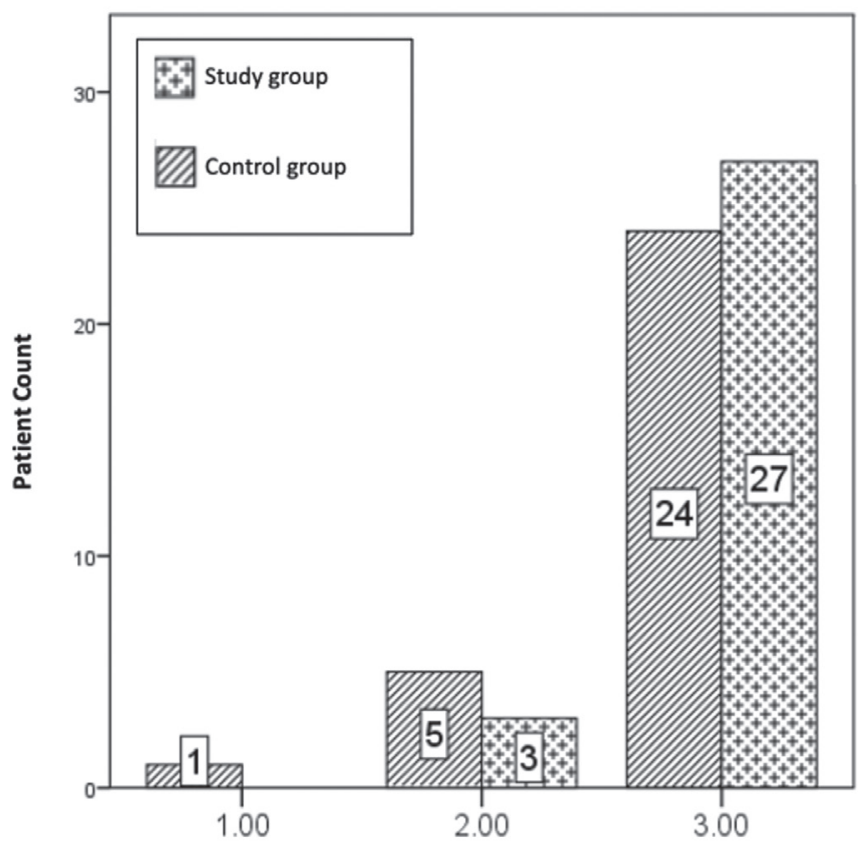

Figure 1. Patient satisfaction according to the trial groups Study group: Para vesical mesh fixation to the fascia; Control group: Para vesical mesh positioning

and three from the study group were only relatively satisfied. Only one patient from the control group was discontented with the operation performed or its corresponding outcomes; the high patient satisfaction could be a result of the improved cure rate and Ba measure, which underscored the subjective clinical benefits gained by the patients from the surgery.

Advanced POP was estimated by the surgeon as that exceeding a $0.5 \mathrm{~L}$ bulging out of the vagina and subsequently and was measured in litres $(0.5 \mathrm{~L}, 0.75 \mathrm{~L}$, and $1.0 \mathrm{~L}$ among others). For the first time, the definition as described earlier is being used and may be useful in defining a special and significant subgroup 
of patients, who are probably prone to higher operative time, complications and recurrent rates. Seven patients were treated for such extreme prolapse and all of them reported good surgical subjective outcomes, even though in one patient, post-op second-degree POP persisted.

The limitations of this study are the relatively small size of the study groups and the short duration of follow-up. Although additional studies on larger groups including a longer duration of follow-up are needed, the results of this study support the view that fixation of anterior arms to the para vesicle fascia is preferable to positioning only. ${ }^{12}$ Furthermore, the results presented here can provide surgeons with valuable decisionmaking advice in selecting the most appropriate surgical procedure using the mini mesh.

\section{CONCLUSIONS}

Mini mesh implantation, with or without anterior arm fixation to the para vesical fascia, led to significant objective and subjective resolution in anterior pelvic floor compartment prolapse. The "anterior mesh arm fixation" resulted in a better POP-Q Ba position.

\section{Authors' contributions}

A.N.K.: Project development, data collection and analysis, and manuscript writing, M.N.: Project development, data analysis, and manuscript writing, J.B.: Project development, and manuscript writing.

\section{Ethics}

Ethics Committee Approval: Retrospective study.

Informed Consent: Retrospective study.

Peer-review: Externally peer-reviewed.

\section{DISCLOSURES}

Financial Disclaimers/Conflict of Interest: MN is affiliated with FEMSelect, Memic, and OORO. ANK and JB report no conflict of interest.

\section{REFERENCES}

1. Olsen AL, Smith VJ, Bergstrom JO, Colling JC, Clark AL. Epidemiology of surgically managed pelvic organ prolapse and urinary incontinence. Obstet Gynecol 1997; 89: 501-506.
2. Cao Q, Chen YS, Ding JX, et al. Long-term treatment outcomes of transvaginal mesh surgery versus anterior-posterior colporrhaphy for pelvic organ prolapse. Aust N Z J Obstet Gynaecol 2013; 53: 7985.

3. Neuman M, Bornstein J. Reconstruction of pelvic organ prolapse: the role of mesh implantation and the need for vaginal hysterectomy. Pelviperineology 2009; 28: 104-108.

4. Petros PE, Ulmsten UI. An integral theory and its method for the diagnosis and management of female urinary incontinence. Scand J Urol Nephrol Suppl 1993; 153: 1-93.

5. Neuman M, Lavy Y. Posterior intra-vaginal slingplasty for the treatment of vaginal apex prolapse: Medium-term results of 140 operations with a novel procedure. Eur J Obstet Gynecol Reprod Biol 2008; 140: 230-233.

6. Neuman M. Sumerova N, Sosnovski V, Bornstein J. Anterior needleguided mesh in advanced pelvic organ prolapse: apical fixation on sacrospinous ligaments. Eur J Obstet Gynecol Reprod Biol 2014; 172 : 120-123.

7. Sumerova N, Neuman M, Krissi H, Pushkar D. The "Pelvic Harness": A skeletonized mesh implant for safe pelvic floor reconstruction. Int Braz J Urol 2016; 42: 507-513.

8. FDA. Urogynecologic Surgical Mesh: Update on the safety and effectiveness of transvaginal placement for pelvic organ prolapse. CDRH 2011; 1-15.

9. FDA. Urogynecologic Surgical Mesh Implants: order to all manufacturers of surgical mesh intended for transvaginal repair of cystocele to stop selling and distributing products immediately. 2019.

10. Persu C, Chapple CR, Cauni V, Gutue S, Geavlete P. Pelvic Organ Prolapse Quantification System (POP-Q) - a new era in pelvic prolapse staging. J Med Life 2011; 4: 75-81.

11. Friedman T, Eslick GD, Dietz HP. Risk factors for prolapse recurrence: systematic review and meta-analysis. Int Urogynecol J 2018; 29: 1321.

12. Weintraub AY, Friedman T, Baumfeld Y, Neuman M, Krissi H. Long term subjective cure rate, urinary tract symptoms and dyspareunia following mesh augmented anterior vaginal wall prolapse repair. Int J Surg 2015; 24: 33-38. 